Reprod. Nutr. Dévelop., 1986, 26 (1 B), 277-278.

\title{
Activités alimentaires et motricité du rumen chez la chèvre et le mouton, pour deux régimes mixtes foin-orge (1)
}

M. FOCANT, M. VANBELLE, Sylvie GODFROID

U.C.L., Place Croix du Sud, 3 ,

B-1348 Louvain-la-Neuve, Belgique.

Summary. Intake of 2 hay-barley rations, rumination and rumen motility have been compared for sheep and goats. Sheep eat more quickly and seem to be able to take in a larger quantity of food in a limited time. For a similar intake, rumination time is longer for sheep. Ruminal contraction frequency is lower for goats during mastication.

Dans certaines conditions tropicales, la digestion des fourrages pauvres serait meilleure chez la chèvre que chez le mouton. Cette différence résulterait du choix alimentaire plus efficace et d'un temps de rétention plus long des aliments dans le réticulo-rumen des caprins (Devendra, 1981), sans doute lié à une motricité plus faible (Ruckebusch et Bost, 1963). De plus, la rumination des ovins, moins efficiente, limiterait leur aptitude à utiliser des fourrages trop fibreux (Welch, 1982).

Matériel et méthodes. Nous avons comparé les activités alimentaires de 4 chèvres Alpine ( 3 ans, $35 \mathrm{~kg}$ ) et de 4 béliers castrés Texel ( 3 ans, $42 \mathrm{~kg}$ ). Les 2 rations utilisées ont compris chacune $40 \mathrm{~g}$ de foin par $\mathrm{kg} \mathrm{P0,75}$ et de l'orge aplatie à raison de $10 \mathrm{~g} / \mathrm{kg} \mathrm{P}^{0,75}$ dans le régime "basse énergie " (BE) et de $35 \mathrm{~g} / \mathrm{kg} \mathrm{P} 0,75$ dans le régime " haute énergie " $(\mathrm{HE})$. Une seule distribution a eu lieu, à $9 \mathrm{~h}$ pour l'orge et $30 \mathrm{~min}$ plus tard pour le foin. La mastication et la motricité du rumen (méthode des "ballonnets ») ont été enregistrées.

Résultats et discussion. La capacité d'ingestion d'un repas unique, dans un temps limité, semble être plus grande chez le mouton que chez la chèvre (tabl. 1 ). Cela se traduit par l'ingestion d'une quantité plus élevée d'orge avec la ration $\mathrm{HE}$, par une durée plus longue du repas principal de foin et par une plus petite durée unitaire d'ingestion (D.U.I.).

Pour des quantités ingérées identiques, le mouton rumine plus longtemps que la chèvre. Conformément aux données de Welch (1982), la durée unitaire de rumination (D.U.R.) est plus grande chez les ovins. Ce pourrait être une compensation de la D.U.I. plus courte. Toutefois, la durée unitaire de mastication est aussi plus longue chez le mouton. Soit la mastication de ce dernier est moins efficiente, soit la taille des particules alimentaires doit être plus petite que chez la chèvre pour pouvoir transiter vers l'omasum. A moins que la plus grande durée de rumination chez le mouton ne soit le reflet d'un besoin plus élevé de Na salivaire (Bell, 1984) pour tamponner le $\mathrm{pH}$ du liquide réticulo-ruminal, plus bas chez les ovins (Focant, 1984).

La fréquence des contractions du sac dorsal du rumen a été exprimée en nombre de contractions par $10 \mathrm{~min}$. En accord avec les résultats de Ruckebusch

(1) Ce travail a été réalisé avec les subsides de I'I.R.S.I.A. 
TABL. 1. - Comparaison des activités alimentaires et de la motricité du rumen de la chèvre $(n=4)$ et du mouton $(n=4)$ pour 2 régimes foin-orge.

\begin{tabular}{|c|c|c|c|c|c|c|}
\hline \multirow{2}{*}{$\frac{\text { Régime }}{\text { Espèce }}$} & \multicolumn{2}{|c|}{ BE } & \multicolumn{2}{|c|}{$\mathrm{HE}$} & \multirow{2}{*}{$\begin{array}{l}\text { Erreur } \\
\text { standard }\end{array}$} & \multirow{2}{*}{$\begin{array}{c}\text { Niveau } \\
\text { de signification }\end{array}$} \\
\hline & Chèvres & Moutons & Chèvres & Moutons & & \\
\hline \multicolumn{7}{|l|}{$\begin{array}{l}\text { Ingestion : } \\
\text { M.S. ingérée }\left(\mathrm{g} / \mathrm{kg}^{0,75} / \text { jour }\right)\end{array}$} \\
\hline $\begin{array}{l}\text { Orge } \\
\text { Foin }\end{array}$ & $\begin{array}{l}10,0 \\
38,9\end{array}$ & $\begin{array}{l}10,0 \\
37,4\end{array}$ & $\begin{array}{l}24,1 \\
33,8\end{array}$ & $\begin{array}{l}34,1 \\
36,0\end{array}$ & $\begin{array}{l}0,42 \\
0,79\end{array}$ & $\begin{array}{l}E^{* * *}, R^{* * *}, E \times R^{* * *} \\
R^{* * *}, E \times R^{*}\end{array}$ \\
\hline $\begin{array}{l}\text { Durée repas princ. foin } \\
\text { (min) }\end{array}$ & 114,0 & 157,7 & 102,7 & 147,5 & 6,49 & $E^{* * *}$ \\
\hline D.U.I. $\left(\min / \mathrm{g}\right.$ M.S. $\left./ \mathrm{kg}^{0,75}\right)$ & & & & & & \\
\hline $\begin{array}{l}\text { Orge } \\
\text { Foin }\end{array}$ & $\begin{array}{l}1,1 \\
5,1\end{array}$ & $\begin{array}{l}0,9 \\
5,2\end{array}$ & $\begin{array}{l}0,9 \\
6,7\end{array}$ & $\begin{array}{l}0,7 \\
5,6\end{array}$ & $\begin{array}{l}0,03 \\
0,27\end{array}$ & $\begin{array}{l}E^{* * *}, R^{* * *} \\
R^{* * *}, E \times R^{*}\end{array}$ \\
\hline Rumination: & & & & & & \\
\hline $\begin{array}{l}\text { Durée de rumination } \\
(\min / 24 \mathrm{~h})\end{array}$ & 478,5 & 530,4 & 465,9 & 636,0 & 11,14 & $E^{* * *}, R^{* * *}, E \times R^{* * *}$ \\
\hline $\begin{array}{l}\text { Durée cycle mérycique } \\
\text { (s) } \\
\left.\text { D.U. (min } / \mathrm{g} \mathrm{NDF} / \mathrm{kg}^{0,75}\right)\end{array}$ & 63,5 & 51,8 & 62,3 & 55,3 & 0,26 & $E^{* *}, R^{*}, E \times R^{* *}$ \\
\hline $\begin{array}{l}\text { Rumination } \\
\text { Mastication }\end{array}$ & $\begin{array}{l}24,3 \\
34,9\end{array}$ & $\begin{array}{l}27,8 \\
38,4\end{array}$ & $\begin{array}{l}22,5 \\
34,1\end{array}$ & $\begin{array}{l}26,0 \\
35,2\end{array}$ & $\begin{array}{l}0,65 \\
0,48\end{array}$ & $\begin{array}{l}E^{* * *}, R^{*} \\
E^{*}, R^{*}\end{array}$ \\
\hline \multicolumn{7}{|c|}{ Motricité du rumen (contractions/10 min): } \\
\hline Repas d'orge & 26,8 & 30,6 & 28,0 & 34,6 & 1,05 & $E^{* * *}, R^{*}$ \\
\hline Repas principal de foin & 28,1 & 30,8 & 28,3 & 30,6 & 0,85 & $\mathrm{E}^{* * *}$ \\
\hline Rumination & 17,3 & 17,6 & 17,1 & 17,9 & 0,38 & 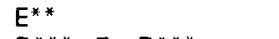 \\
\hline Périodes de repos & 16,9 & 15,4 & 14,9 & 15,5 & 0,39 & $R^{* * *}, E \times R^{* * *}$ \\
\hline
\end{tabular}

$\mathrm{BE}: 10 \mathrm{~g}$ orge $/ \mathrm{kg}^{0.75}+40 \mathrm{~g}$ foin $/ \mathrm{kg}^{0,75} ; \mathrm{HE}: 35 \mathrm{~g}$ orge $/ \mathrm{kg}^{0,75}+40 \mathrm{~g}$ foin $/ \mathrm{kg}^{0,75}$. $\mathrm{E}=$ espèce, $\mathrm{R}=$ régime, $\mathrm{E} \times \mathrm{R}=$ interaction espèce $\times$ régime. D.U.I. = Durée unitaire d'ingestion ; $\mathrm{D}$. $U .=$ Durée unitaire. ${ }^{*}=\mathrm{P}<0,05 ;{ }^{* *}=\mathrm{P}<0,01 ;{ }^{* *}=\mathrm{P}<0,001$.

et Bost (1963), elle est moindre chez la chèvre, tant pendant l'ingestion de l'orge et du foin que pendant la rumination. Cette activité motrice plus faible du rumen peut être mise en relation avec d'une part la vitesse d'ingestion plus lente, et d'autre part la durée moyenne plus longue des cycles méryciques. En dehors des périodes de mastication, la motricité du sac dorsal du rumen n'est pas différente pour les 2 espèces.

En conc/usion, la chèvre semble rassasiée plus vite que le mouton, elle rumine moins longtemps et son rumen se contracte à une moindre fréquence pendant la mastication. Des études complémentaires sont toutefois nécessaires pour élucider les causes physiologiques de ces différences et leurs implications nutritionnelles.

Bell F. R., 1984. Neuroendocrine control of ingestive behavior in cattle. Can. J. anim. Sci, 64 (Suppl.), 326-327.

Dévendra C., 1981. The utilisation of forages from cassava, pigeon pea, leucaena and groundnut by goats and sheep in Malaysia, 338-345. In Morand-Fehr P., Bourbouze A., De Simiane M., Nutrition and systems of goat feeding, Vol. 1, I.N.R.A.-ITOVIC, Symp. Int., Tours, France, 12/15 mai 1981.

Focant M., 1984. Comportement alimentaire, rumination, fermentations réticulo-ruminales et acides gras volatils plasmatiques, comparés chez la chèvre et le mouton ; influence du régime. Reprod. Nutr. Dévelop., 24, 239-250.

Ruckebusch Y., Bost J., 1963. Etude comparée de la motricité du réticulum et du comportement alimentaire, chez les ovins et caprins en " stabulation " Rev. Méd. vét., 114, 184-195.

Welch J. G., 1982. Rumination, particle size and passage from the rumen. J. anim. Sci., 54, 885-894. 